TRANSACTIONS OF THE

AMERICAN MATHEMATICAL SOCIETY

Volume 352, Number 6, Pages 2569-2579

S 0002-9947(00)02398-9

Article electronically published on March 7, 2000

\title{
ON SYZYGIES OF ABELIAN VARIETIES
}

\author{
ELENA RUBEI
}

\begin{abstract}
In this paper we prove the following result: Let $X$ be a complex torus and $M$ a normally generated line bundle on $X$; then, for every $p \geq 0$, the line bundle $M^{p+1}$ satisfies Property $N_{p}$ of Green-Lazarsfeld.
\end{abstract}

\section{INTRODUCTION}

In this paper we prove a new result on syzygies of abelian varieties; precisely, the problem we are considering is the following: let $X$ be a complex torus, $L$ a very ample line bundle on $X$ and $\varphi_{L}$ the associated map; we are concerned with the degree of the equations defining $\varphi_{L}(X)$, the degree of the syzygies among them and the degree of higher syzygies. In particular, here we examine the case where $L=M^{l}$ where $M$ is a normally generated line bundle.

To review precisely the statements of the known results on syzygies of abelian varieties and to formulate precisely our theorem, we have to recall Green-Lazarsfeld's definition of Property $N_{p}$ (see [Gr1], G-L], Gr2], [Laz2], E-L]): let $Y$ be a smooth complex projective variety of dimension $n$ and let $L$ be a very ample line bundle on $Y$ defining an embedding $Y \subset \mathbf{P}=\mathbf{P}\left(H^{0}(Y, L)^{*}\right)$; set $S=S(L)=\operatorname{Sym}^{*} H^{0}(L)$, the homogeneous coordinate ring of the projective space $\mathbf{P}$, and consider the graded $S$-module $G=G(L)=\bigoplus_{d} H^{0}\left(Y, L^{d}\right)$. Let $E_{*}$ be a minimal graded free resolution of $G$ (that is, an exact sequence with $E_{i}$ free $S$-modules and such that the matrices of homogenous polynomials giving the maps $E_{i} \longrightarrow E_{i-1}$ has no nonzero constant entries); the line bundle $L$ satisfies Property $N_{p}(p \in \mathbf{N})$ if and only if

$$
\begin{aligned}
& E_{0}=S, \\
& E_{i}=\bigoplus S(-i-1) \quad \text { for } 1 \leq i \leq p .
\end{aligned}
$$

(Thus $L$ satisfies Property $N_{0}$ if and only if $Y \subset \mathbf{P}\left(H^{0}(L)^{*}\right)$ is projectively normal, that is, $L$ is normally generated; $L$ satisfies Property $N_{1}$ if and only if $L$ satisfies Property $N_{0}$ and the homogeneous ideal $I$ of $Y \subset \mathbf{P}\left(H^{0}(L)^{*}\right)$ is generated by quadrics; $L$ satisfies Property $N_{2}$ if and only if $L$ satisfies Property $N_{1}$ and the module of syzygies among quadratic generators $Q_{i} \in I$ is spanned by relations of the form $\sum L_{i} Q_{i}=0$, where $L_{i}$ are linear polynomials; and so on.)

In 1966 Mumford proved that, if $M$ is an ample line bundle on a complex torus $X$ and $l \geq 4$, then the ideal of $\varphi_{M^{l}}(X)$ is generated by quadrics (Mum2]) and in

Received by the editors November 30, 1997 and, in revised form, March 29, 1998.

2000 Mathematics Subject Classification. Primary 14K05.

Key words and phrases. Abelian varieties, syzygies.

This research was carried through in the realm of the AGE Project HCMERBCHRXCT940557 and of the ex-40 MURST Program "Geometria algebrica". 
1978 Sekiguchi proved a similiar result for $l=3$ ([Se]). In 1989 Kempf proved again and generalized these results in [Ke] precisely the following theorem holds (part $c$ ) was proved by Lange and Birkenhake using Kempf's proof; see 7.4.1, [L-B]):

Theorem 1 (Mumford-Sekiguchi-Kempf). Let $X$ be a complex torus. If $A$ is an ample line bundle on $X$ we denote $I(A)$ the ideal of $\varphi_{A}(X)$. Let $M$ be an ample line bundle on $X$;

a) if $l \geq 4$, the ideal $I\left(M^{l}\right)$ is generated by forms of degree 2 ,

b) let $l=3$, the ideal $I\left(M^{3}\right)$ is generated by forms of degrees 2 and 3 ,

c) (Lange-Birkenhake) let $l=2$; if $M^{2}$ is normally generated, then the ideal $I\left(M^{2}\right)$ is generated by forms of degrees 2,3 and 4 .

In 1984 Green proved that if $X$ is a Riemann surface of genus $g$ and $L$ is a holomorphic line bundle on $X$ of degree $2 g+1+p$, then $L$ satisfies Property $N_{p}$ (see Gr1 and Gr2]). Thus, if $M$ is an ample line bundle on an elliptic curve, then $M^{p+3}$ satisfies Property $N_{p}$ and in Laz2 Lazarsfeld formulated the following conjecture:

Conjecture 2 (Lazarsfeld). If $M$ is an ample line bundle on a complex torus, then, for every $p \geq 0$, the line bundle $M^{p+3}$ satisfies Property $\left(N_{p}\right)$.

In 1989 Kempf proved a weaker result (see $[\mathrm{Ke}]$ ):

Theorem 3 (Kempf). Let $M$ be an ample line bundle on a complex torus $X$. If $l \geq 4$, then $M^{l}$ satisfies Property $N_{\left[\frac{l-2}{2}\right]}$.

In 1993 Ein and Lazarsfeld proved the following theorem (see [E-L]):

Theorem 4 (Ein-Lazarsfeld). Let $Y$ be a smooth complex projective variety of dimension $n$; let $A$ be a very ample line bundle on $Y$, and $B$ a numerically effective line bundle on $Y$; then $K_{Y} \otimes A^{n+1+p} \otimes B$ satisfies Property $N_{p}$.

If $(Y, A, B) \neq\left(\mathbf{P}^{n}, \mathcal{O}_{\mathbf{P}^{n}}(1), \mathcal{O}_{\mathbf{P}^{n}}\right)$ and $p \geq 1$, then $K_{Y} \otimes A^{n+p} \otimes B$ satisfies Property $N_{p}$.

Thus, if $M$ is a very ample line bundle on a complex torus of dimension $n$, then $M^{n+p}$ satisfies Property $N_{p}$.

In this paper, using the ideas of Kempf's paper [Ke], by a patient adaptation, we prove another theorem on syzygies of abelian varieties:

Theorem 5. If $M$ is a normally generated line bundle on a complex torus $X$, then, for every $p \geq 0$, the line bundle $M^{p+1}$ satisfies Property $N_{p}$.

Since there is a result on normal generation of primitive line bundles (Lazarsfeld's theorem on projective normality of $(1, d)$-abelian surfaces; see [Laz1]), Theorem 5 may actually be useful (see Remark 18).

Notation and Definitions. We collect here some notation and standard definitions that we will use throughout the paper.

- $\varphi_{L}$. If $L$ is a line bundle on a complex manifold $Y, \varphi_{L}$ is the rational map associated to $L$.

- A line bundle $L$ on a complex manifold $Y$ is called normally generated if it is very ample and $\varphi_{L}(Y)$ is projectively normal. We have that $L$ is normally generated if and only if it is ample and the natural maps $S^{n} H^{0}(Y, L) \longrightarrow$ $H^{0}\left(Y, L^{n}\right)$ are surjective for all $n \geq 2$ (see [Mum1], p. 38 and [L-B], Chapter $7, \S 3)$. 
If $X$ is a complex torus of dimension $g$, then

- $\boldsymbol{t}_{\boldsymbol{x}}$ is the translation on $X$ by the point $x$;

- $\hat{\boldsymbol{X}}$ is the dual complex torus of $X$; it is isomorphic to $\operatorname{Pic}^{0}(X)$;

- $\mathcal{P}$ denotes the Poincaré bundle on $X \times \hat{X}$;

- $\phi_{L}$ is the homomorphism $X \longrightarrow \hat{X}, x \mapsto t_{x}^{*} L \otimes L^{-1}$, where $L$ is a line bundle on $X$;

- $\boldsymbol{K}(\boldsymbol{L})$ is the kernel of $\phi_{L}$; it depends only on $H$, the first Chern class of $L$, thus we denote $K(L)$ also by $\boldsymbol{K}(\boldsymbol{H})$; if $L$ is nondegenerate, then $K(L)$ is a finite group isomorphic to $\left(\mathbf{Z} / d_{1} \oplus \ldots \oplus \mathbf{Z} / d_{g}\right)^{2}$ with $d_{i} \mid d_{i+1}$; we say that $L$ is of type $\left(d_{1}, \ldots, d_{g}\right)$;

- $\boldsymbol{W} \cdot \boldsymbol{W}^{\prime}$ : if $W$ is a vector subspace of $H^{0}(X, E)$ and $W^{\prime}$ is a vector subspace of $H^{0}\left(X, E^{\prime}\right)\left(E\right.$ and $E^{\prime}$ line bundles on $\left.X\right), W \cdot W^{\prime}$ is the image of $W \otimes W^{\prime}$ under the multiplication map; we often omit $\cdot$

- $\pi$ : if we have a product of tori, we use the notation: $\pi_{i}$ is the projection on the $i$ th factor and $\pi$. is the projection on $\cdot$

\section{Some RECALLS}

First we recall Mumford's lemma (see Mum1] or [L-B], Chapter 7, Lemma 3.3) and the following remark and proposition.

Lemma 6 (Mumford). Let $A$ and $B$ be two ample line bundles on a complex torus $X$. For every nonempty open subset $U$ of $\hat{X}$, we have

$$
\sum_{P \in U} H^{0}(X, A \otimes P) \cdot H^{0}\left(X, B \otimes P^{-1}\right)=H^{0}(X, A \otimes B) .
$$

As Kempf observed in $[\mathrm{Ke}$, Mumford's lemma can be interpreted in this way: a linear functional $\lambda$ on $H^{0}(A \otimes B)$ is determined by the family $\left\{\lambda_{P}\right\}_{P \in U}$, where $\lambda_{P}$ is the linear functional on $H^{0}(X, A \otimes P) \otimes H^{0}\left(X, B \otimes P^{-1}\right)$ given by the composition of the multiplication with $\lambda$.

Remark 7 (see Gr2]). Let $V$ be a complex vector space of dimension $r+1$, let $S=\bigoplus_{q \geq 0} S y m^{q}(V)$ and $G=\bigoplus_{q} G_{q}$ a finitely generated graded $S$-module. Let

$$
0 \longrightarrow E_{n} \longrightarrow E_{n-1} \longrightarrow \ldots \longrightarrow E_{0} \longrightarrow G \longrightarrow 0
$$

be a minimal free resolution of $G$, that is, an exact sequence with $E_{i}$ free $S$-modules and such that the matrices of homogenous polynomials giving the maps $E_{i} \longrightarrow E_{i-1}$ has no nonzero constant entries. Write $E_{p}=\bigoplus_{q}\left(B_{p, q} \otimes S(-q)\right)$ with $B_{p, q}$ vector spaces on $\mathbf{C}$. See $\mathbf{C}$ as the $S$-module $S / \bigoplus_{q \geq 1} \operatorname{Sym}^{q}(V)$. Then

$$
\operatorname{Tor}_{p}^{S}(G, \mathbf{C})_{q} \simeq B_{p, q} .
$$

Proposition 8 (Koizumi [Ko]). Let $A$ and $A^{\prime}$ be two algebraically equivalent ample line bundles on a complex torus $X$. The multiplication map $H^{0}\left(X, A^{m}\right) \otimes$ $H^{0}\left(X, A^{\prime n}\right) \longrightarrow H^{0}\left(X, A^{m} \otimes A^{\prime n}\right)$ is surjective for all $m \geq 3$ and $n \geq 2$.

Now we recall some facts, definitions and propositions of Kempf's paper [Ke].

Definition 9 (Kempf). For any $A_{i}$ 's ample line bundles on a complex torus $X$, let $K\left(A_{1}\right)=H^{0}\left(X, A_{1}\right)$ and, for $n>1$, define $K\left(A_{1}, \ldots, A_{n}\right)$ inductively by the following exact sequence:

$$
0 \rightarrow K\left(A_{1}, \ldots, A_{n}\right) \rightarrow K\left(A_{1}, A_{3}, \ldots, A_{n}\right) \otimes H^{0}\left(X, A_{2}\right) \rightarrow K\left(A_{1} \otimes A_{2}, A_{3}, \ldots, A_{n}\right) .
$$


To follow completely Kempf's notations, we denote $K\left(A_{1}, A_{2}\right)$ by $R\left(A_{1}, A_{2}\right)$ $\left(=\operatorname{ker}\left(H^{0}\left(A_{1}\right) \otimes H^{0}\left(A_{2}\right) \longrightarrow H^{0}\left(A_{1} \otimes A_{2}\right)\right)\right.$ ).

In the sequel $K\left(A_{1}, A_{3}, \ldots, A_{n}\right) \cdot H^{0}\left(X, A_{2}\right)$ will denote the image of the multiplication map $K\left(A_{1}, A_{3}, \ldots, A_{n}\right) \otimes H^{0}\left(X, A_{2}\right) \longrightarrow K\left(A_{1} \otimes A_{2}, A_{3}, \ldots, A_{n}\right)\left(\subset H^{0}\left(A_{1} \otimes\right.\right.$ $\left.\left.A_{2}\right) \otimes H^{0}\left(A_{3}\right) \otimes \ldots \otimes H^{0}\left(A_{n}\right)\right)$; we often omit $\cdot$

Notation 10 (Kempf). In the remainder of this section, following [Ke], we use the following notation: let $X$ be a complex torus of dimension $g$; fix an ample line bundle $M$ on $X ; l_{i}, i \in \mathbf{N}$, will denote positive integers and $L_{i}$ will denote a line bundle algebraically equivalent to $M^{l_{i}}$.

Observe that, if $A$ is a line bundle on $X$, since $H^{0}\left(\left.\left(\pi_{X}^{*} A \otimes \mathcal{P}\right)\right|_{X \times\{P\}}\right)=$ $H^{0}(A \otimes P)$ is of constant dimension $\forall P \in \hat{X}$, then the sheaf $\pi_{\hat{X} *}\left(\pi_{X}^{*} A \otimes \mathcal{P}\right)$ on $\hat{X}$ is locally free and its fibre over $P \in \hat{X}$ is $H^{0}(A \otimes P)$, by Grauert's Theorem (see [Ha] Theorem 12.9 Chapter 3). Analogously the sheaf $\pi_{\hat{X} *}\left(\pi_{X}^{*} A \otimes \mathcal{P}^{-1}\right)$ on $\hat{X}$ is locally free and its fibre over $P \in \hat{X}$ is $H^{0}\left(A \otimes P^{-1}\right)$.

Consider the following map:

$$
\pi_{\hat{X} *}\left(\pi_{X}^{*} L_{1} \otimes \mathcal{P}\right) \otimes \pi_{\hat{X} *}\left(\pi_{X}^{*} L_{2} \otimes \mathcal{P}^{-1}\right) \longrightarrow H^{0}\left(X, L_{1} \otimes L_{2}\right) \otimes_{\mathbf{C}} \mathcal{O}_{\hat{X}}
$$

(given by the composition of the maps

$$
\pi_{\hat{X} *}\left(\pi_{X}^{*} L_{1} \otimes \mathcal{P}\right) \otimes \pi_{\hat{X} *}\left(\pi_{X}^{*} L_{2} \otimes \mathcal{P}^{-1}\right) \longrightarrow \pi_{\hat{X} *} \pi_{X}^{*}\left(L_{1} \otimes L_{2}\right)
$$

and

$$
\left.\pi_{\hat{X} *} \pi_{X}^{*}\left(L_{1} \otimes L_{2}\right) \longrightarrow H^{0}\left(X, L_{1} \otimes L_{2}\right) \otimes_{\mathbf{C}} \mathcal{O}_{\hat{X}} \cdot\right)
$$

This map induces a map:

$$
m: H^{0}\left(X, L_{1} \otimes L_{2}\right)^{\vee} \longrightarrow H^{0}\left(\hat{X},\left(\pi_{\hat{X} *}\left(\pi_{X}^{*} L_{1} \otimes \mathcal{P}\right) \otimes \pi_{\hat{X} *}\left(\pi_{X}^{*} L_{2} \otimes \mathcal{P}^{-1}\right)\right)^{\vee}\right) .
$$

Proposition 11 (Kempf). i) The map

$$
m: H^{0}\left(X, L_{1} \otimes L_{2}\right)^{\vee} \longrightarrow H^{0}\left(\hat{X},\left(\pi_{\hat{X} *}\left(\pi_{X}^{*} L_{1} \otimes \mathcal{P}\right) \otimes \pi_{\hat{X} *}\left(\pi_{X}^{*} L_{2} \otimes \mathcal{P}^{-1}\right)\right)^{\vee}\right)
$$

is an isomorphism.

ii) $H^{i}\left(\hat{X},\left(\pi_{\hat{X} *}\left(\pi_{X}^{*} L_{1} \otimes \mathcal{P}\right) \otimes \pi_{\hat{X} *}\left(\pi_{X}^{*} L_{2} \otimes \mathcal{P}^{-1}\right)\right)^{\vee}\right)=0$ for $i \geq 1$.

Arguing exactly as in Proposition 4 of $[\mathrm{Ke}$, we have:

Proposition 12 (Kempf). If $H^{0}\left(L_{1} \otimes P\right) \otimes H^{0}\left(L_{3}\right) \longrightarrow H^{0}\left(L_{1} \otimes P \otimes L_{3}\right)$ is surjective $\forall P \in P i c^{0}(X)$ and $H^{0}\left(L_{1} \otimes L_{2}\right) \otimes H^{0}\left(L_{3}\right) \longrightarrow H^{0}\left(L_{1} \otimes L_{2} \otimes L_{3}\right)$ is surjective, then

$$
\sum_{P \in P i c^{0}(X)} R\left(L_{1} \otimes P, L_{3}\right) \cdot H^{0}\left(L_{2} \otimes P^{-1}\right)=R\left(L_{1} \otimes L_{2}, L_{3}\right) .
$$

We reproduce the proof here for later use.

Proof. One inclusion is obvious:

$$
\sum_{P \in \hat{X}} R\left(L_{1} \otimes P, L_{3}\right) \cdot H^{0}\left(L_{2} \otimes P^{-1}\right) \subset R\left(L_{1} \otimes L_{2}, L_{3}\right) .
$$

We want to show the other one. It suffices to show that $\left(\sum_{P \in P i c^{0}(X)} R\left(L_{1} \otimes P, L_{3}\right)\right.$. $\left.H^{0}\left(L_{2} \otimes P^{-1}\right)\right)^{\perp}$ in $\left(H^{0}\left(L_{1} \otimes L_{2}\right) H^{0}\left(L_{3}\right)\right)^{\vee}$ is contained in $R\left(L_{1} \otimes L_{2}, L_{3}\right)^{\perp}$. 
For every $P \in \hat{X}$, we have the following commutative diagram:

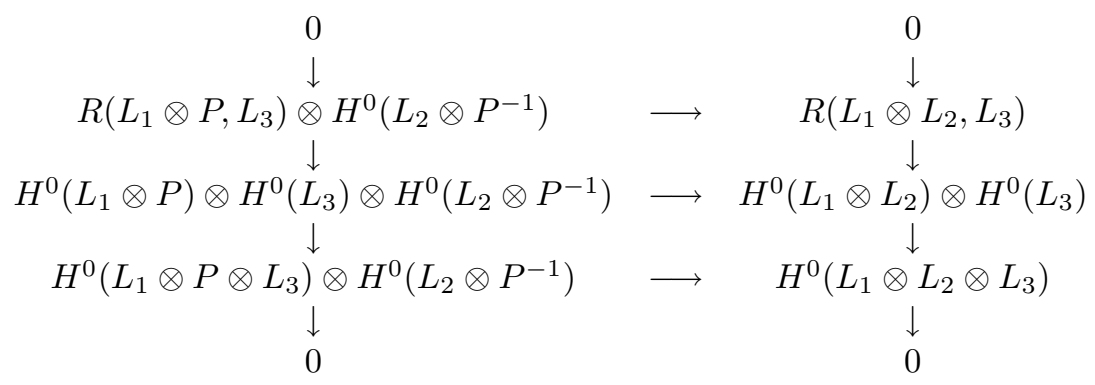

The columns are exact by the hypotheses.

To see that $\left(\sum_{P \in P i c^{0}(X)} R\left(L_{1} \otimes P, L_{3}\right) \cdot H^{0}\left(L_{2} \otimes P^{-1}\right)\right)^{\perp}$ in $\left(H^{0}\left(L_{1} \otimes L_{2}\right) \otimes\right.$ $\left.H^{0}\left(L_{3}\right)\right)^{\vee}$ is contained in $R\left(L_{1} \otimes L_{2}, L_{3}\right)^{\perp}$ is equivalent to seeing that any element of $\left(H^{0}\left(L_{1} \otimes L_{2}\right) \otimes H^{0}\left(L_{3}\right)\right)^{\vee}$ inducing a linear form on $H^{0}\left(L_{1} \otimes L_{3} \otimes P\right) \otimes H^{0}\left(L_{2} \otimes P^{-1}\right)$ induces a linear form on $H^{0}\left(L_{1} \otimes L_{2} \otimes L_{3}\right)$ and this is true by Proposition[1]

Definition 13 (Kempf). Let $S$ be a graded ring and $G$ a finitely generated graded $S$-module and $k=S / \bigoplus_{n \geq 1} S_{n}$. Define $d(G)=\min \{j \mid G$ is generated by the union of the $G_{d}$ 's for $\left.d \leq j\right\}$ (thus $d(G)$ is the smallest number such that $G \otimes_{S} k$ is zero in degree $>d(G)$ ).

Define $T^{1}(G)=\operatorname{ker}\left(G(-1) \otimes_{k} S_{1} \longrightarrow G\right)$.

Define $T^{j}(G)=T^{j-1}\left(T^{1}(G)\right)$ and $T^{0}(G)=G$.

Define $d^{j}(G)=d\left(T^{j}(G)\right)$.

Lemma 14 (Kempf). Let $S$ be a graded ring and $G$ a finitely generated graded $S$-module and $k=S / \bigoplus_{n \geq 1} S_{n}$. If $q>i-j+d\left(T^{j}(G)\right)$ for all $0 \leq j \leq i$, then $\operatorname{Tor}_{i}^{S}(G, k)$ is zero in degree $q$.

\section{TWO NEW LEMMAS}

To prove Theorem 5 we need the following two lemmas.

Lemma 15. Let $A$ and $A^{\prime}$ be two algebraically equivalent normally generated line bundles on a complex torus $X$ and $m, n \in \mathbf{N}$. If $m \geq 2$ and $n \geq 1$, then

$$
H^{0}\left(A^{m}\right) \cdot H^{0}\left(A^{\prime n}\right)=H^{0}\left(A^{m} \otimes A^{\prime n}\right) .
$$

Proof. Observe that the set $U:=\left\{P \in P i c^{0}(X)\right.$ s.t. $H^{0}\left(A \otimes P^{-1}\right) H^{0}\left(A^{\prime n}\right)=$ $\left.H^{0}\left(A \otimes A^{\prime n} \otimes P^{-1}\right)\right\}$ is nonempty, since $A$ and $A^{\prime}$ are normally generated, and open, since, as we have already observed, for any line bundle $L$ on $X$, the vector spaces $H^{0}(L \otimes P) P \in \hat{X}$ form a vector bundle on $\hat{X}$.

Applying twice Mumford's Lemma, we have

$$
\begin{aligned}
& H^{0}\left(A^{m}\right) H^{0}\left(A^{\prime n}\right)=\sum_{P \in U} H^{0}\left(A^{m-1} \otimes P\right) H^{0}\left(A \otimes P^{-1}\right) H^{0}\left(A^{\prime n}\right) \\
& =\sum_{P \in U} H^{0}\left(A^{m-1} \otimes P\right) H^{0}\left(A \otimes A^{\prime n} \otimes P^{-1}\right)=H^{0}\left(A^{m} \otimes A^{\prime n}\right)
\end{aligned}
$$

for $m \geq 2$ and $n \geq 1$.

Lemma 16. Let $A, A^{\prime}$ and $A^{\prime \prime}$ be three algebraically equivalent normally generated line bundles on a complex torus $X$ and $\alpha, \beta, \gamma \in \mathbf{N}$. If we are in one of the following three cases: 
1) $\alpha \geq 2, \beta \geq 2, \gamma \geq 2$,

2) $\alpha \geq 3, \beta=1, \alpha+\gamma \geq 5$,

3) $\alpha \geq 3, \gamma=1, \alpha+\beta \geq 5$,

then

$$
R\left(A^{\alpha}, A^{\prime \beta}\right) \cdot H^{0}\left(A^{\prime \prime \gamma}\right)=R\left(A^{\alpha} \otimes A^{\prime \prime \gamma}, A^{\prime \beta}\right) .
$$

Proof. Let $\alpha, \beta, \gamma, l \in \mathbf{N}$ and $\beta, l, \alpha-l \geq 1$.

If

$$
H^{0}\left(A^{\alpha-l} \otimes P\right) \otimes H^{0}\left(A^{\prime \beta}\right) \longrightarrow H^{0}\left(A^{\alpha-l} \otimes P \otimes A^{\prime \beta}\right)
$$

for all $P \in P i c^{0}(X)$, and

$$
H^{0}\left(A^{\alpha}\right) \otimes H^{0}\left(A^{\prime \beta}\right) \longrightarrow H^{0}\left(A^{\alpha} \otimes A^{\prime \beta}\right)
$$

are surjective (we call this condition (a)), then, by Proposition 12 we have

$$
R\left(A^{\alpha}, A^{\prime \beta}\right) H^{0}\left(A^{\prime \prime \gamma}\right)=\sum_{P \in P i c^{0}(X)} R\left(A^{\alpha-l} \otimes P, A^{\prime \beta}\right) H^{0}\left(A^{l} \otimes P^{-1}\right) H^{0}\left(A^{\prime \prime \gamma}\right) .
$$

If

$$
H^{0}\left(A^{l} \otimes P^{-1}\right) \otimes H^{0}\left(A^{\prime \prime \gamma}\right) \longrightarrow H^{0}\left(A^{l} \otimes P^{-1} \otimes A^{\prime \prime \gamma}\right)
$$

for all $P \in P i c^{0}(X)$, is surjective (we call this condition (b)), then we have

$$
\begin{aligned}
& \sum_{P \in P i c^{0}(X)} R\left(A^{\alpha-l} \otimes P, A^{\prime \beta}\right) H^{0}\left(A^{l} \otimes P^{-1}\right) H^{0}\left(A^{\prime \prime \gamma}\right) \\
& =\sum_{P \in P i c^{0}(X)} R\left(A^{\alpha-l} \otimes P, A^{\prime \beta}\right) H^{0}\left(A^{l} \otimes A^{\prime \prime \gamma} \otimes P^{-1}\right) .
\end{aligned}
$$

If

$$
H^{0}\left(A^{\alpha-l} \otimes P\right) \otimes H^{0}\left(A^{\prime \beta}\right) \longrightarrow H^{0}\left(A^{\alpha-l} \otimes P \otimes A^{\prime \beta}\right)
$$

for all $P \in P i c^{0}(X)$, and

$$
H^{0}\left(A^{\alpha} \otimes A^{\prime \prime \gamma}\right) \otimes H^{0}\left(A^{\prime \beta}\right) \longrightarrow H^{0}\left(A^{\alpha} \otimes A^{\prime \prime \gamma} \otimes A^{\prime \beta}\right)
$$

are surjective (we call this condition (c)), then, by Proposition 12, we have

$$
\sum_{P \in P i c^{0}(X)} R\left(A^{\alpha-l} \otimes P, A^{\prime \beta}\right) H^{0}\left(A^{l} \otimes A^{\prime \prime \gamma} \otimes P^{-1}\right)=R\left(A^{\alpha} \otimes A^{\prime \prime \gamma}, A^{\prime \beta}\right) .
$$

By Lemma 15 if we are in one of the following four cases:

1) $\alpha \geq 2, \beta \geq 2, \gamma \geq 2, l=1$,

2) $\alpha \geq 4, \beta=1, \gamma=1, l=2$,

2') $\alpha \geq 3, \beta=1, \gamma \geq 2, \alpha+\gamma \geq 5, l=1$

3) $\alpha \geq 3, \gamma=1, \alpha+\beta \geq 5, l=2$,

then (a), (b) and (c) hold. Thus we conclude the proof. 


\section{THE PROOF OF THEOREM [5] AND TWO REMARKS}

The crucial step to prove Theorem [5] is the following proposition, which is analogous to Theorem 17 in [Ke].

Proposition 17. Let $M$ be a normally generated line bundle on a complex torus $X$. We again use Notation 10 , that is the $l_{i}$ 's, $i \in \mathbf{N}$, denote positive integers and $L_{i}$ denotes a line bundle algebraically equivalent to $M^{l_{i}}$.

a) Let $m \geq 3$. If $l_{1} \geq m-1, \ldots, l_{m} \geq m-1$, then $K\left(L_{1}, L_{3}, \ldots, L_{m}\right) \otimes$ $H^{0}\left(X, L_{2}\right) \longrightarrow K\left(L_{1} \otimes L_{2}, L_{3}, \ldots, L_{m}\right)$ is surjective.

b) Let $m \geq 1$. If $l_{1} \geq m, \ldots, l_{m} \geq m$, then $K\left(L_{1}, \ldots, L_{m}\right)$ form a vector bundle on the appropriate component of $\operatorname{Pic}(X)^{m}$.

c) Let $m \geq 4$. If $l_{1} \geq m-2, l_{2} \geq 1$ and $l_{3} \geq m-1, \ldots, l_{m} \geq m-1$, then $K\left(L_{1} \otimes L_{2}, L_{3}, \ldots, L_{m}\right)=\sum_{P \in \hat{X}} K\left(L_{1} \otimes P, L_{3}, \ldots, L_{m}\right) \cdot H^{0}\left(L_{2} \otimes P^{-1}\right)$.

d) Let $m \geq 2$. Let $l_{1} \geq 2 m-1, l_{2} \geq 1$, and, if $m \geq 3, l_{3} \geq m, \ldots, l_{m} \geq m$ and suppose the family of vector spaces $K\left(L_{1} \otimes P, L_{3}, \ldots, L_{m}\right), P \in \hat{X}$, forms a vector bundle on $\hat{X}$, we call the corresponding sheaf $\mathcal{F}_{m-1}$. Then we have

i) an isomorphism

$$
K\left(L_{1} \otimes L_{2}, L_{3}, \ldots, L_{m}\right)^{\vee}=H^{0}\left(\hat{X},\left(\mathcal{F}_{m-1} \otimes \pi_{\hat{X} *}\left(\pi_{X}^{*} L_{2} \otimes \mathcal{P}^{-1}\right)\right)^{\vee}\right) .
$$

ii) $H^{i}\left(\hat{X},\left(\mathcal{F}_{m-1} \otimes \pi_{\hat{X} *}\left(\pi_{X}^{*} L_{2} \otimes \mathcal{P}^{-1}\right)\right)^{\vee}\right)=0$ if $i \geq 1$.

Proof. Consider the following four statements depending on the natural number $m$ :

- Statement $A(m)$ : If $l_{1} \geq m-1, \ldots, l_{m} \geq m-1$, then $K\left(L_{1}, L_{3}, \ldots, L_{m}\right) \otimes$ $H^{0}\left(X, L_{2}\right) \longrightarrow K\left(L_{1} \otimes L_{2}, L_{3}, \ldots, L_{m}\right)$ is surjective.

- Statement $B(m)$ : If $l_{1} \geq m, \ldots, l_{m} \geq m$, then $K\left(L_{1}, \ldots, L_{m}\right)$ form a vector bundle on the appropriate component of $\operatorname{Pic}(X)^{m}$.

- Statement $C(m)$ : If $l_{1} \geq m-2, l_{2} \geq 1$, and, if $m \geq 3, l_{3} \geq m-1, \ldots, l_{m} \geq$ $m-1$, then $K\left(L_{1} \otimes L_{2}, L_{3}, \ldots, L_{m}\right)=\sum_{P \in \hat{X}} K\left(L_{1} \otimes P, L_{3}, \ldots, L_{m}\right) \cdot H^{0}\left(L_{2} \otimes P^{-1}\right)$.

- Statement $D(m)$ : Let $l_{1} \geq 2 m-1, l_{2} \geq 1$, and, if $m \geq 3, l_{3} \geq m, \ldots, l_{m} \geq m$ and suppose the family of vector spaces $K\left(L_{1} \otimes P, L_{3}, \ldots, L_{m}\right), P \in \hat{X}$, forms a vector bundle on $\hat{X}$, we call the corresponding sheaf $\mathcal{F}_{m-1}$; then we have

i) an isomorphism

$$
K\left(L_{1} \otimes L_{2}, L_{3}, \ldots, L_{m}\right)^{\vee}=H^{0}\left(\hat{X},\left(\mathcal{F}_{m-1} \otimes \pi_{\hat{X} *}\left(\pi_{X}^{*} L_{2} \otimes \mathcal{P}^{-1}\right)\right)^{\vee}\right),
$$

ii) $H^{i}\left(\hat{X},\left(\mathcal{F}_{m-1} \otimes \pi_{\hat{X} *}\left(\pi_{X}^{*} L_{2} \otimes \mathcal{P}^{-1}\right)\right)^{\vee}\right)=0$ if $i \geq 1$.

We know that $A(3), B(1), B(2), D(2)$ are true $(A(3)$ is true by Lemma 16, $B(2)$ by Lemma 15, $D(2)$ by Proposition 11).

We will prove the following four implications:

- $A(m-1)$ and $B(m-2) \Rightarrow B(m-1)$ for $m \geq 3$.

- $A(m-1), B(m-2)$ and $D(m-1) \Rightarrow D(m)$ for $m \geq 4$ and $B(1)$ and $D(2) \Rightarrow$ $D(3)$.

- $A(m-1), B(m-2)$ and $D(m-1) \Rightarrow C(m)$ for $m \geq 3$.

- $C(m) \Rightarrow A(m)$ for $m \geq 3$.

By the second implication also $D(3)$ holds; using the four implications, one can prove by induction that $A(m), B(m-1)$ and $D(m)$ are true for $m \geq 3$ and conclude.

Thus let us prove the four implications.

- $A(m-1)$ and $B(m-2) \Rightarrow B(m-1)$ for $m \geq 3$ : obvious.

- $A(m-1), B(m-2)$ and $D(m-1) \Rightarrow D(m)$ for $m \geq 4$ and $B(1)$ and $D(2) \Rightarrow$ $D(3)$; it can be proved in an analogous way as Proposition 9 in $\mathrm{Ke}$; more precisely: 
consider line bundles $L_{1}, \ldots, L_{m}$ with the hypotheses of $D(m)$, thus $l_{1} \geq 2 m-1$, $l_{2} \geq 1$, and, if $m \geq 3, l_{3} \geq m, \ldots, l_{m} \geq m$

Let $m \geq 4$. Since $l_{1}, l_{3}, \ldots, l_{m} \geq m-2$, by $A(m-1)$ we have the following exact sequence:

$$
\begin{aligned}
& 0 \longrightarrow K\left(L_{1} \otimes P, L_{3}, \ldots, L_{m}\right) \otimes H^{0}\left(L_{2} \otimes P^{-1}\right) \\
& \quad \longrightarrow K\left(L_{1} \otimes P, L_{4}, \ldots, L_{m}\right) \otimes H^{0}\left(L_{3}\right) \otimes H^{0}\left(L_{2} \otimes P^{-1}\right) \\
& \longrightarrow K\left(L_{1} \otimes L_{3} \otimes P, L_{4}, \ldots L_{m}\right) \otimes H^{0}\left(L_{2} \otimes P^{-1}\right) \longrightarrow 0
\end{aligned}
$$

observe that also if $m=3$ this exact sequence holds, by Proposition 8

The above sequence gives an exact sequence

$$
\begin{aligned}
0 \longrightarrow\left(\mathcal{F}_{m-2}^{\prime} \otimes \pi_{\hat{X} *}\left(\pi_{X}^{*} L_{2} \otimes \mathcal{P}^{-1}\right)\right)^{\vee} \\
\longrightarrow\left(\mathcal{F}_{m-2} \otimes H^{0}\left(L_{3}\right) \otimes \pi_{\hat{X} *}\left(\pi_{X}^{*} L_{2} \otimes \mathcal{P}^{-1}\right)\right)^{\vee} \\
\longrightarrow\left(\mathcal{F}_{m-1} \otimes \pi_{\hat{X} *}\left(\pi_{X}^{*} L_{2} \otimes \mathcal{P}^{-1}\right)\right)^{\vee} \longrightarrow 0,
\end{aligned}
$$

with $\mathcal{F}_{m-2}$ the sheaf corresponding to the bundle whose fibre over $P \in \hat{X}$ is $K\left(L_{1} \otimes P, L_{4}, \ldots, L_{m}\right)$ and $\mathcal{F}_{m-2}^{\prime}$ the sheaf corresponding to the bundle whose fibre over $P \in \hat{X}$ is $K\left(L_{1} \otimes L_{3} \otimes P, L_{4}, \ldots, L_{m}\right)$ (they are bundles by $B(m-2$ ), in fact, the hypotheses of $B(m-2)$ for them, that is $l_{1}, l_{4}, \ldots, l_{m} \geq m-2$ and $l_{1}+l_{3}, l_{4}, \ldots, l_{m} \geq m-2$, hold).

We take the cohomology sequence associated to the above exact sequence. By ii) of $D(m-1)$ we have ii) of $D(m)$.

Then we obtain

$$
\begin{aligned}
0 & \longrightarrow H^{0}\left(\left(\mathcal{F}_{m-2}^{\prime} \otimes \pi_{\hat{X} *}\left(\pi_{X}^{*} L_{2} \otimes \mathcal{P}^{-1}\right)\right)^{\vee}\right) \\
& \longrightarrow H^{0}\left(\left(\mathcal{F}_{m-2} \otimes H^{0}\left(L_{3}\right) \otimes \pi_{\hat{X} *}\left(\pi_{X}^{*} L_{2} \otimes \mathcal{P}^{-1}\right)\right)^{\vee}\right) \\
& \longrightarrow H^{0}\left(\left(\mathcal{F}_{m-1} \otimes \pi_{\hat{X} *}\left(\pi_{X}^{*} L_{2} \otimes \mathcal{P}^{-1}\right)\right)^{\vee}\right) \longrightarrow 0,
\end{aligned}
$$

which is equal to

$$
\begin{aligned}
0 & \longrightarrow K\left(L_{1} \otimes L_{2} \otimes L_{3}, L_{4}, \ldots, L_{m}\right)^{\vee} \\
& \longrightarrow\left(K\left(L_{1} \otimes L_{2}, L_{4}, \ldots, L_{m}\right) H^{0}\left(L_{3}\right)\right)^{\vee} \\
& \longrightarrow H^{0}\left(\left(\mathcal{F}_{m-1} \otimes \pi_{\hat{X} *}\left(\pi_{X}^{*} L_{2} \otimes \mathcal{P}^{-1}\right)\right)^{\vee}\right) \longrightarrow 0,
\end{aligned}
$$

by $D(m-1)$ (we have to verify that $l_{1}+l_{3} \geq 2(m-1)-1, l_{4}, \ldots, l_{m} \geq m-1, l_{2} \geq 1$ and $l_{1} \geq 2(m-1)-1$ and that it is actually true).

By $A(m-1)$ we have

$$
\begin{aligned}
0 & \longrightarrow K\left(L_{1} \otimes L_{2} \otimes L_{3}, L_{4}, \ldots, L_{m}\right)^{\vee} \\
& \longrightarrow\left(K\left(L_{1} \otimes L_{2}, L_{4}, \ldots, L_{m}\right) H^{0}\left(L_{3}\right)\right)^{\vee} \\
& \longrightarrow K\left(L_{1} \otimes L_{2}, L_{3}, L_{4}, \ldots, L_{m}\right)^{\vee} \longrightarrow 0,
\end{aligned}
$$

(to apply $A(m-1)$ we have to verify that $l_{1}+l_{2} \geq m-2, l_{3}, . ., l_{m} \geq m-2$ and it is true).

Thus $H^{0}\left(\left(\mathcal{F}_{m-1} \otimes \pi_{\hat{X} *}\left(\pi_{X}^{*} L_{2} \otimes \mathcal{P}^{-1}\right)\right)^{\vee}\right)=K\left(L_{1} \otimes L_{2}, L_{3}, \ldots, L_{m}\right)^{\vee}$.

- $A(m-1), B(m-2)$ and $D(m-1) \Rightarrow C(m)$ for $m \geq 3$ : this implication can be proved in an analogous way as in Proposition 12.

- $C(m) \Rightarrow A(m)$ for $m \geq 3$ : it can be proved in an analogous way as in Theorem 5 in $\left[\mathrm{Ke}\right.$; more precisely, let $l_{1}, \ldots, l_{m} \geq m-1$; write $L_{1}=L_{1}^{\prime} \otimes M$ with $L_{1}^{\prime}$ 
algebraically equivalent to $M^{l_{1}-1}$. We have

$$
K\left(L_{1} \otimes L_{2}, L_{3}, \ldots, L_{m}\right)=\sum_{P \in P i c^{0}(X)} K\left(L_{1}^{\prime} \otimes P, L_{3}, \ldots, L_{m}\right) H^{0}\left(M \otimes L_{2} \otimes P^{-1}\right)
$$

if

$$
l_{1}-1 \geq m-2, \quad 1+l_{2} \geq 1, \quad l_{3}, \ldots, l_{m} \geq m-1,
$$

by $C(m)$.

We have

$$
\begin{aligned}
& \sum_{P \in P i c^{0}(X)} K\left(L_{1}^{\prime} \otimes P, L_{3}, \ldots, L_{m}\right) H^{0}\left(M \otimes L_{2} \otimes P^{-1}\right) \\
& \quad=\sum_{P \in P i c^{0}(X)} K\left(L_{1}^{\prime} \otimes P, L_{3}, \ldots, L_{m}\right) H^{0}\left(M \otimes P^{-1}\right) H^{0}\left(L_{2}\right)
\end{aligned}
$$

if

$$
l_{2} \geq 2
$$

by Lemma 15 .

We have

$\sum_{P \in P i c^{0}(X)} K\left(L_{1}^{\prime} \otimes P, L_{3}, \ldots, L_{m}\right) H^{0}\left(M \otimes P^{-1}\right) H^{0}\left(L_{2}\right)=K\left(L_{1}, L_{3}, \ldots, L_{m}\right) H^{0}\left(L_{2}\right)$

if

$\left(*_{3}\right)$

$$
l_{1}-1 \geq m-2, \quad 1 \geq 1, \quad l_{3}, \ldots, l_{m} \geq m-1
$$

by $C(m)$.

$\left(*_{1}\right),\left(*_{2}\right),\left(*_{3}\right)$ are true, thus we conclude the proof of this implication.

Now we are ready to prove Theorem 5 .

Proof of Theorem 5. For any line bundle $L$ on $X$ we denote $G(L)=\bigoplus_{n} H^{0}\left(L^{n}\right)$, a module over the ring $S(L)=\operatorname{Sym}^{0}(L)$.

By Remark 7, we have to prove that $\operatorname{Tor}_{i}^{S\left(M^{p+1}\right)}\left(G\left(M^{p+1}\right), \mathbf{C}\right)$ is purely of degree $i+1$ for $1 \leq i \leq p$.

Thus we have to prove that $\operatorname{Tor}_{i}^{S\left(M^{p+1}\right)}\left(G\left(M^{p+1}\right), \mathbf{C}\right)$ is zero in degree $\geq i+2$ for $1 \leq i \leq p$.

By Lemma 14, it is sufficient to prove that

$$
i+2>i-j+d\left(T^{j}\left(G\left(M^{p+1}\right)\right)\right)
$$

for $0 \leq j \leq i$ and $1 \leq i \leq p$, (we use Definition 13), that is,

$$
j+1 \geq d\left(T^{j}\left(G\left(M^{p+1}\right)\right)\right)
$$

for $0 \leq j \leq i$ and $1 \leq i \leq p$, that is,

$$
j+1 \geq d\left(T^{j}\left(G\left(M^{p+1}\right)\right)\right)
$$

for $0 \leq j \leq p$.

Observe that

$$
T^{j}\left(G\left(M^{p+1}\right)\right)=\bigoplus_{n} K(M^{(p+1)(n-j)}, \overbrace{M^{p+1}, \ldots, M^{p+1}}^{J}) ;
$$


then using Proposition 17 part a) with $m-1=p+1$ we have that, if $p \geq j$, then $T^{j}\left(G\left(M^{p+1}\right)\right)$ is generated by $K\left(M^{p+1}, \ldots, M^{p+1}\right)$ (where $M^{p+1}$ repeats $j+1$ times), that is by the part of degree $n$ with $n-j=1$ that is $n=j+1$; thus $d\left(T^{j}\left(G\left(M^{p+1}\right)\right)\right)=j+1$ and we conclude.

Remark 18. Let $X_{i}$ be a complex torus and $L_{i}$ a line bundle on $X_{i}$ for $i=1,2$; one can easily see that, if $L_{i}$ satisfies Property $N_{0}$ for $i=1,2$, then the line bundle $\pi_{1}^{*} L_{1} \otimes \pi_{2}^{*} L_{2}$ on $X_{1} \times X_{2}$ satisfies Property $N_{0}$ and if $L_{i}$ satisfies Property $N_{1}$ for $i=1,2$, then the line bundle $\pi_{1}^{*} L_{1} \otimes \pi_{2}^{*} L_{2}$ on $X_{1} \times X_{2}$ satisfies Property $N_{1}$.

In Laz1, Lazarsfeld proved that, if $X$ is a complex torus of dimension $2, L$ is an ample line bundle of type $(1, d)$ on $X,|L|$ has no fixed components and $\varphi_{L}$ is birational onto its image, then $\varphi_{L}(X)$ is projectively normal for $d$ odd $\geq 7$ and $d$ even $\geq 14$.

Thus, for instance, if $d \in \mathbf{N}$ is even and $\geq 14$, one can deduce from Theorem 5 and Lazarsfeld's Theorem that, if $\left(X, c_{1}(L)\right)$ is generic in the moduli space of polarized abelian threefolds of type $(2,4,2 d)$, the line bundle $L$ on the complex torus $X$ satisfies Property $N_{1}$; in fact, one can consider an elliptic curve $E$ with an ample line bundle $A$ of type (4) and an abelian surface $S$ with a very ample line bundle $M$ of type $(1, d)$ satisfying the hypotheses of Lazarsfeld's Theorem (it exists by Reider's Theorem, which claims that, if $M$ is an ample line bundle of type $(1, d)$ with $d \geq 5$ on a complex torus $X$ of dimension 2 , then $M$ is very ample if and only if there is no elliptic curve $C$ on $X$ with $(C \cdot L)=2$; thus generically an ample line bundle of type $(1, d)$ with $d \geq 5$ on a complex torus $X$ of dimension 2 is very ample; see [Re or [L-B] Chapter $10, \S 4$ ); the line bundle $A$ satisfies Property $N_{1}$ by Theorem 1 and the line bundle $M^{2}$ satisfies Property $N_{1}$ by Lazarsfeld's Theorem and Theorem 5 thus, considering the product $(E, A) \times\left(S, M^{2}\right)$, we conclude.

More generally, one can prove analogously the following statement: let $d_{i} \in \mathbf{N}$ $i=1, \ldots, g, d_{i} \mid d_{i+1}, 1<s+1 \leq t<g, d_{1}=\ldots=d_{s}=1, d_{s+1}, \ldots, d_{t} \geq 2$, $d_{t+1}, \ldots, d_{g} \in\{d \in \mathbf{N} \mid d \geq 7$ odd or $d \geq 14$ even $\}$; if $g-t \geq s$, then, if $\left(X, c_{1}(L)\right)$ is generic in the moduli space of polarized abelian varieties of type $\left(2 d_{1}, \ldots \ldots, 2 d_{g}\right)$, the line bundle $L$ on the complex torus $X$ satisfies Property $N_{1}$.

Remark 19. One can conjecture that, if $M$ is an ample line bundle on a complex torus $X$ and $M^{s}$ satisfies Property $N_{k}$, then $M^{s+p}$ satisfies Property $N_{k+p}$.

Observe that for $s=3$ and $k=0$ this is Lazarsfeld's conjecture and for $s=1$ and $k=0$ this is Theorem 5

\section{ACKNOWLEDGMENTS}

I thank Professor F. Catanese for some useful discussions.

\section{REFERENCES}

[E-L] L. Ein R. Lazarsfeld, Syzygies and Koszul cohomology of smooth projective varieties of arbitrary dimension, Invent. Math. 111 (1993) no. 1, 51-67. MR 93m:13006

[Gr1] M. Green, Koszul cohomology and the geometry of projective varieties I, II, J. Differential Geom. 19 (1984), 125-171; J. Differential Geom. 20 (1984), 279-289. MR 85e:14022, MR 86j:14011

[Gr2] M. Green, Koszul cohomology and geometry, in: (M. Cornalba et al. eds), Lectures on Riemann Surfaces, World Scientific Press (1989). MR 91k:14012

[G-L] M. Green and R. Lazarsfeld, On the projective normality of complete linear series on an algebraic curve, Invent. Math. 83 (1986), 73-90. MR 87g:14022 
[Ha] R. Hartshorne, Algebraic Geometry, Grad. Texts Math. 52, Springer-Verlag, BerlinHeidelberg-New York, 1977. MR 57:3116

[Ke] G. Kempf, Projective cooridinate rings of abelian varieties, in: Algebraic Analysis, Geometry and Number Theory, edited by I.J. Igusa, The John Hopkins Press (1989), 225-236. MR 98m:14047

[Ko] S. Koizumi, Theta relations and projective normality of abelian varieties, Amer. J. Math. 98 (1976), 865-889. MR 58:702

[L-B] H. Lange and Ch. Birkenhake, Complex Abelian Varieties, Springer-Verlag, 1992. MR 94j:14001

[Laz1] R. Lazarsfeld, Projectivité normale des surfaces abéliannes, redigé par O. Debarre. prépublication No. 14 Europroj - C.I.M.P.A., Nice, 1990.

[Laz2] R. Lazarsfeld, A sampling of vector bundle techniques in the study of linear series, in: (M. Cornalba et al. (eds), Lectures on Riemann Surfaces, World Scientific Press (1989), 500-559. MR 92f:14006

[Mum1] D. Mumford, Varieties defined by quadratic equations in: Questioni sulle varietà algebriche, Corsi C.I.M.E., Edizioni Cremonese, Roma, (1969), 29-100. MR 44:209

[Mum2] D. Mumford, On equations defining abelian varieties, Invent. Math. 1 (1966), 287-354. MR 34:4269

[Re] I. Reider, Vector bundles of rank 2 and linear systems on algebraic surfaces, Ann. of Math., 127 (1988), 309-316. MR 89e:14038

[Se] T. Sekiguchi, On normal generation by a line bundle on an abelian variety, Proc. Japan Acad. 54, Ser A (1978), 185-188. MR 80c:14026

Dipartimento di Matematica, Università di Pisa, via F. Buonarroti 2, Pisa (PI) c.A.P. 56127, ITALIA

E-mail address: rubei@mail.dm.unipi.it 